\title{
Superconducting contacts to a monolayer
}

\section{semiconductor}

\author{
M. Ramezani, ${ }^{\dagger} \ddagger$ I. Correa Sampaio, ${ }^{\dagger}$ K. Watanabe,,${ }^{\text {II }}$ T. Taniguchi, ${ }^{\S}$ C. \\ Schönenberger, ${ }^{\dagger, \ddagger}$ and A. Baumgartner, $, \dagger, \ddagger$ \\ $\dagger$ Department of Physics, University of Basel, CH-4056, Basel, Switzerland \\ $\ddagger$ Swiss Nanoscience Institute, University of Basel, CH-4056, Basel, Switzerland \\ IIResearch Center for Functional Materials, National Institute for Material Science, 1-1 Namiki, \\ Tsukuba, 305-0044, Japan \\ §International Center for Materials Nanoarchitectonics, National Institute for Materials Science, \\ 1-1 Namiki, Tsukuba 305-0044, Japan \\ E-mail: andreas.baumgartner@unibas.ch
}

\begin{abstract}
We demonstrate superconducting vertical interconnect access (VIA) contacts to a monolayer of molybdenum disulfide $\left(\mathrm{MoS}_{2}\right)$, a layered semiconductor with highly relevant electronic and optical properties. As a contact material we use MoRe, a superconductor with a high critical magnetic field and high critical temperature. The electron transport is mostly dominated by a single superconductor/normal conductor junction with a clear superconductor gap. In addition, we find $\mathrm{MoS}_{2}$ regions that are strongly coupled to the superconductor, resulting in resonant Andreev tunneling and junction dependent gap characteristics, suggesting a superconducting proximity effect. Magnetoresistance measurements show that the bandstructure and the high intrinsic carrier mobility remain intact in the bulk of the $\mathrm{MoS}_{2}$. This type of VIA contact is applicable to a large variety of layered materials and superconducting
\end{abstract}


contacts, opening up a path to monolayer semiconductors as a platform for superconducting hybrid devices.

keywords: TMDC, van der Waals heterostructure, $M o S_{2}$, monolayer semiconductor, superconducting contacts, superconducting proximity effect 
Semiconductors combined with superconducting metals have become a most fruitful field for applications and fundamental research, from gate tunable superconducting qubits, ${ }^{1}$ thermoelectrics, ${ }^{2,3}$ to prospective Majorana bound states, ${ }^{4,5}$ or sources of entangled electron pairs. ${ }^{6-8}$ These experiments were mainly developed based on one-dimensional (1D) nanowires. To obtain more flexible platforms and scalable architectures, recent efforts focused on two-dimensional (2D) semiconductors. ${ }^{9-13}$ However, the number of materials suitable for superconducting hybrids is rather limited. Potentially ideal and ultimately thin semiconductors with a large variety of properties can be found among transition metal dichalcogenides (TMDCs) grown in stacked atomically thin layers. TMDCs often exhibit a broad variety of interesting optical and electronic properties, ${ }^{14-16}$ for example the valley degree of freedom, potentially useful as qubits, ${ }^{17,18}$ strong electron-electron ${ }^{19,20}$ and spin-orbit interactions, ${ }^{20}$ or crystals with topologically non-trivial bandstructures. ${ }^{21,22}$ One promising material is the semiconductor $\mathrm{MoS}_{2}$, with a relatively high mobility and large mean free path, allowing for gate-defined nanostructures, ${ }^{23-25}$ which would make $\mathrm{MoS}_{2}$ an ideal platform to combine with superconducting elements.

$\mathrm{MoS}_{2}$ was used as tunnel barrier between superconductors in vertical heterostructures ${ }^{26,27}$ and showed signs of intrinsic superconductivity ${ }^{28}$ and of a ferromagnetic phase at low electron densities. ${ }^{29}$ However, to exploit the intrinsic properties of $\mathrm{MoS}_{2}$ and to fabricate in-situ gate tunable superconducting hybrid structures, direct superconducting contacts in lateral devices are required. Such contacts are difficult to fabricate due to the formation of Schottky barriers, ${ }^{21,30,31}$ material degradation $^{32}$ and fabrication residues when using standard fabrication methods. ${ }^{24,33,34}$ Less conventional edge contacts were also found problematic recently. ${ }^{35}$

Here, we report vertical access interconnect (VIA) contacts ${ }^{36}$ to monolayer $\mathrm{MoS}_{2}$ with the superconductor MoRe as contact material. We demonstrate a clear superconducting gap in the transport characteristics, including the magnetic field and temperature dependence, and features suggesting stronger superconductor-semiconductor couplings, forming the basis for superconducting proximity effects and bound states. In addition, we show that this fabrication method retains the intrinsic $\mathrm{MoS}_{2}$ bulk properties, including a large electron mobility and sequentially occupied 
spin-orbit split bands. ${ }^{23}$

Figure 1(a) shows an optical microscopy image of the presented device, and a schematic of a single VIA contact. The $\mathrm{MoS}_{2}$ is fully encapsulated by exfoliated hexagonal boron nitride (hBN), ensuring minimal contamination of the bulk materials, while the following assembly process allows the fabrication of pristine material interfaces and contacts:

1) vertical access: using electron beam lithography (EBL), VIA areas with a radius of $200 \mathrm{~nm}$ are defined on the designated top hBN flake ( $\sim 40 \mathrm{~nm}$ thickness $)$ on a $\mathrm{Si} / \mathrm{SiO}_{2}$ wafer, and etched completely open by reactive ion etching with a 20:5:5 sccm $\mathrm{SF}_{6}: \mathrm{O}_{2}:$ Ar mixture at 25 mTorr chamber pressure and $50 \mathrm{~W}$ RF power.

2) VIA metalization: in a second EBL step, a slightly larger area with the VIA in the center is defined for mechanical anchoring to the top $\mathrm{hBN}$. We then deposit the type II superconductor MoRe (bulk critical temperature $T_{\mathrm{c}} \approx 6-10 \mathrm{~K}$, (second) critical magnetic field $B_{\mathrm{c}} \approx 8-9 \mathrm{~T}^{37,38}$ ) using sputter techniques. As the optimal film thickness we find $10 \mathrm{~nm}$ plus the top hBN thickness.

3) Stacking of layers: the wafer with the VIA structure is transferred to an inert gas (nitrogen) glove box, where the top hBN layer with the metalized VIAs is picked up from the substrate using a polycarbonate (PC) stamp and an $\mathrm{hBN}$ helper layer, and then used to pick up consecutively a monolayer $\mathrm{MoS}_{2}$ flake, a bottom hBN flake ( $\sim 25 \mathrm{~nm}$ thickness), and a multilayer graphene (MLG) flake serving as backgate.

4) Finish: the stack is then deposited onto a $\mathrm{Si} / \mathrm{SiO}_{2}$ wafer, where macroscopic $\mathrm{Ti} / \mathrm{Au}(10 / 50 \mathrm{~nm})$ leads to the VIAs are fabricated using EBL. The sample is then annealed at $350^{\circ} \mathrm{C}$ for $30 \mathrm{~min}$, in a vacuum chamber with a constant flow of forming gas.

Using gold as VIA material, this fabrication process yields $>80 \%$ of the contacts with twoterminal resistance-area products smaller than $200 \mathrm{k} \Omega \mu \mathrm{m}^{2}$ at $T=1.7 \mathrm{~K}$ at a backgate voltage of $V_{\mathrm{BG}}=10 \mathrm{~V}$. This yield is reduced to roughly $50 \%$ when using MoRe, possibly due to a material loss during the pick-up procedure. In the presented device, only half of the contacts show resis- 

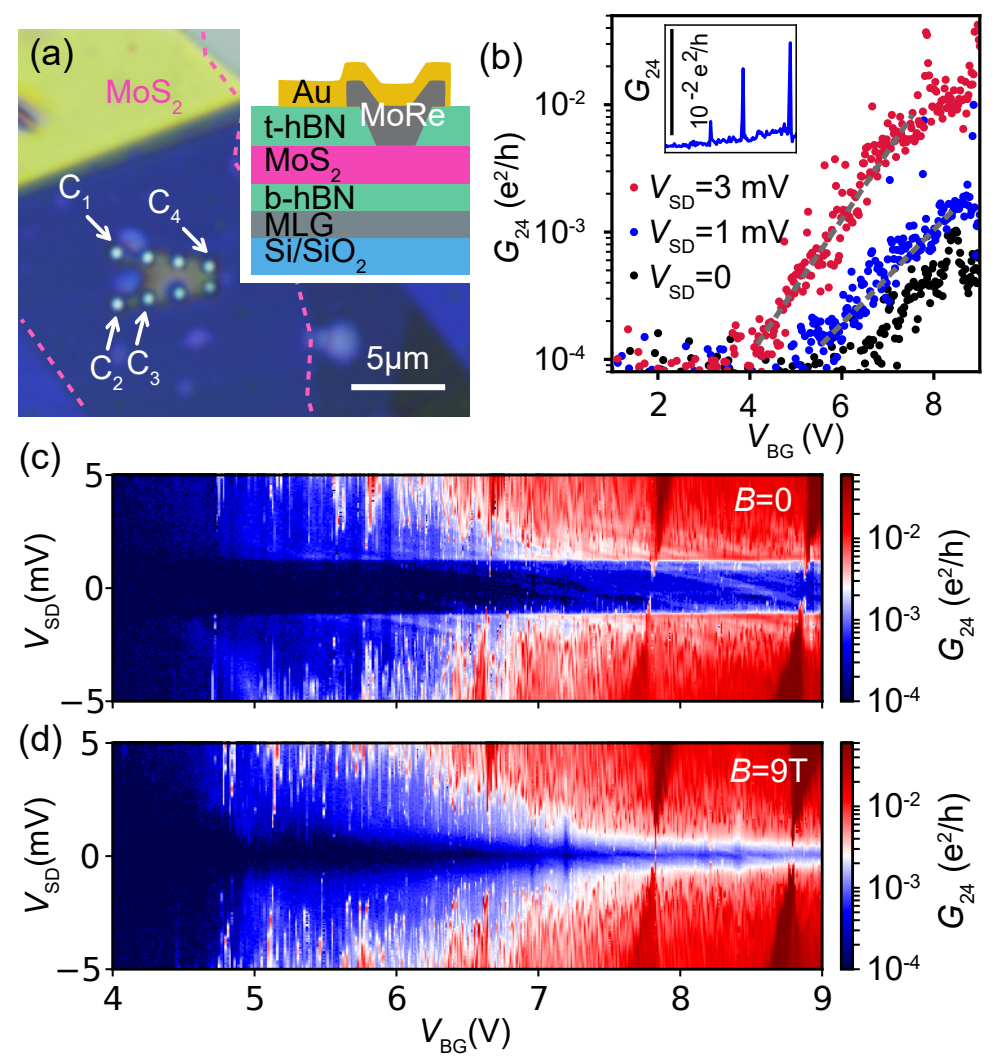

Figure 1: (a) Optical microscopy image of the $\mathrm{MoS}_{2}$ heterostructure, with MoRe VIA contacts pointed out by white arrows. Inset: schematic of a VIA contact, with t-hBN and b-hBN denoting the top and bottom hBN layers, respectively. (b) Differential conductance $G_{24}$ as a function of $V_{\mathrm{BG}}$ for a series of $V_{\mathrm{SD}}$ on a logarithmic scale, and as an inset for $V_{\mathrm{SD}}=1 \mathrm{mV}$ on a linear scale. (c) $G_{24}$ vs. $V_{\mathrm{BG}}$ and $V_{\mathrm{SD}}$ at $B=0$ and (d) at $B=9 \mathrm{~T}$.

tances lower than $200 \mathrm{k} \Omega \mu \mathrm{m}^{2}$, which we use for the experiments and are labeled from $\mathrm{C}_{1}$ to $\mathrm{C}_{4}$ in Fig. 1(a). The two terminal differential conductances $G_{j k}=\mathrm{d} I_{j} / \mathrm{d} V_{k}$ we obtain by measuring the current variation in the grounded contact $\mathrm{C}_{j}$ while applying a modulated bias voltage $V_{\mathrm{SD}}$ to contact $\mathrm{C}_{k}$ using standard lock-in techniques. The experiments were performed in a dilution refrigerator at $\sim 60 \mathrm{mK}$, while for higher temperatures, we used a variable temperature insert (VTI) with a base temperature of $\sim 1.7 \mathrm{~K}$. In addition, we apply an external magnetic field $B$ perpendicular to the substrate.

In Fig. 1(b), the differential conductance $G_{24}$ is plotted as a function of the backgate voltage $V_{\mathrm{BG}}$ for several bias voltages $V_{\mathrm{SD}}$. Increasing $V_{\mathrm{BG}}$ results in an exponential increase in $G_{24}$, starting from a pinch-off voltage of $V_{\mathrm{BG}} \approx 6 \mathrm{~V}$ for $V_{\mathrm{SD}}=0$. This value is offset towards smaller values for 
larger bias voltages, a first indication for an energy gap. However, as seen in the inset of Fig. 1(b), in this regime we find very sharp peaks in $G$, consistent with Coulomb blockade (CB) effects. We note that an increase in $V_{\mathrm{BG}}$ not only changes the charge carrier density in the $\mathrm{MoS}_{2}$, but also the Schottky barrier at the metal-semiconductor interface and disorder induced charge islands. To demonstrate a superconducting energy gap and to distinguish it from other effects like $\mathrm{CB}$, we plot in Fig. 1(c) the conductance $G_{24}$ vs. $V_{\mathrm{SD}}$ over a large range of $V_{\mathrm{BG}}$ at $B=0$, while Fig. 1(d) shows the same experiment at $B=9 \mathrm{~T}$. At $B=0$, independent of the gate voltage, one clearly finds a strongly suppressed conductance for roughly $\left|V_{\mathrm{SD}}\right|<1.2 \mathrm{mV}$, a gap size consistent with literature values for the superconducting energy gap of MoRe. ${ }^{39}$ The conductance is suppressed by a factor $\sim 8$ between the large and the zero bias values at $V_{\mathrm{BG}} \approx 6 \mathrm{~V}$, and by a factor of $\sim 15$ near $V_{\mathrm{BG}}=8 \mathrm{~V}$. We note that such a sharp gap is only observable if a tunnel barrier is formed between the semiconducting $\mathrm{MoS}_{2}$ and the superconducting region, at least at one contact. The discrete features inside the gap are probably not Andreev bound states, ${ }^{40,41}$ but rather originate from gate-modulated conductance features in the bulk $\mathrm{MoS}_{2}$. At $\left|V_{\mathrm{SD}}\right|>1.2 \mathrm{mV}$, we find a strong modulation of $G$, which we interpret as several Coulomb blockaded regions. These resonances suggest that there is significant disorder near some of these contacts, so that we can think of this device as an $\mathrm{MoS}_{2}$ region, incoherently coupled to the contacts by two normal-superconductor (N/S) junctions. The reason for one junction, namely the less transparent one, dominating the transport characteristics is that the junction with the higher transmission has a reduced resistance in the energy gap due to Andreev reflection, in which two electrons are transferred to $\mathrm{S}$ to form a Cooper pair. At $B=9 \mathrm{~T}$, the superconducting gap is reduced, as discussed below in detail, but we now find that the gap becomes gate dependent. While in short semiconducting nanowires a gate tunable proximity effect was reported, ${ }^{42,43}$ we tentatively attribute our finding to a gate independent superconducting energy gap convoluted with a gate tunable $\mathrm{MoS}_{2}$ conductance.

We investigate the gap in the transport characteristics and the field dependence in more detail in Fig. 2 for the same contact pair. Figures 2(a)-(c) show higher resolution conductance maps for a smaller $V_{\mathrm{BG}}$ interval, for the magnetic fields $B=0, B=5 \mathrm{~T}$ and $B=9 \mathrm{~T}$, respectively. The figures 

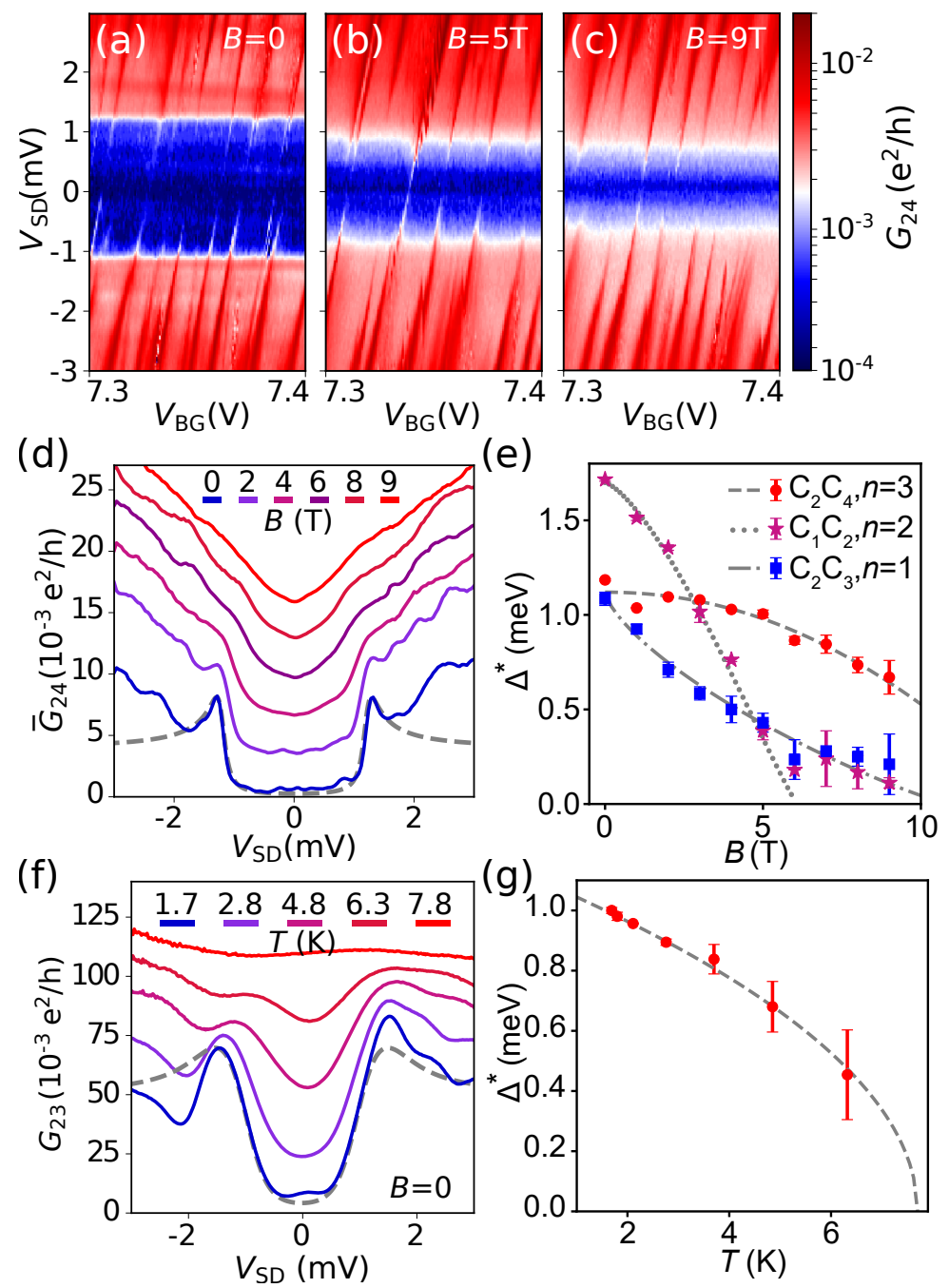

Figure 2: (a) Differential conductance $G_{24}$ as a function of the bias $V_{\mathrm{SD}}$ and the backgate voltage $V_{\mathrm{BG}}$ at $B=0$, (b) $B=5 \mathrm{~T}$, and (c) $B=9 \mathrm{~T}$. (d) $G_{24}$ averaged over a $V_{\mathrm{BG}}$ interval of $0.5 \mathrm{~V}$ plotted vs. $V_{\mathrm{SD}}$ for the indicated magnetic fields, with the curves offset for clarity. (e) Superconducting energy gap $\Delta^{*}$ as a function of $B$ for different contact pairs. $\Delta^{*}$ is extracted from the inflection points in the curves of Fig. 2(d) [red disks], from Fig. 3 [purple stars] and from an additional data set discussed in the supplemental data, Fig. S1 [blue rectangles]. (f) $G_{23}$ vs. $V_{\text {SD }}$ recorded at the indicated temperatures $T$. (g) $\Delta^{*}$ vs. $T$ extracted from the data in (f). All theoretical curves (dashed and dotted lines) are discussed in the text.

show a very clear gap in the conductance map, which is systematically reduced with increasing magnetic field, independently of the sharp resonances. The positions of the latter are unaffected by the gap, so that we can attribute them to resonances in the $\mathrm{MoS}_{2}$, for example due to CB. To extract the energy gap, we plot $G_{24}$ in Fig. 2(d), averaged over a gate voltage interval of $0.5 \mathrm{~V}$ for each $V_{\mathrm{SD}}$ value, for a series of perpendicular magnetic fields. These curves show how the energy gap closes 
with increasing $B$. The curve at $B=0$ can be fitted well using the model by Blonder, Tinkham and Klapwjik (BTK) ${ }^{44}$ including an additional broadening parameter, ${ }^{45}$ as shown in Fig. 2(d) by a gray dashed line. The fit parameters are consistent with a weakly transmitting barrier in a single N/S junction. At larger fields, the extracted parameters become ambiguous due to a strong broadening of the curves. As a measure for the superconducting energy gap $\Delta^{*}$, we therefore plot in Fig. 2(e) the average of the low-bias inflection points of each curve (red dots). For $B=0$, we find $\Delta_{0}^{*} \approx 1.2 \mathrm{meV}$, in good agreement with bulk MoRe $\mathrm{e}^{26,39}$ and one $\mathrm{S} / \mathrm{N}$ junction dominating the transport. The field dependence of $\Delta^{*}$ is well described by standard theory of superconductivity for pair breaking impurities in a metal with a mean free path shorter than the superconducting coherence lengths. For the corresponding self-consistency equations we use $\Delta^{*}(\alpha)=\hat{\Delta}(\alpha)[1-$ $\left.(\alpha / \hat{\Delta}(\alpha))^{2 / 3}\right]^{3 / 2}$, with $\Delta^{*}(\alpha)$ the energy gap in the excitation spectrum and $\hat{\Delta}$ the order parameter determined numerically from $\ln \left(\hat{\Delta}(\alpha) / \Delta_{0}\right)=-\pi \alpha / 4 \hat{\Delta}(\alpha)$ for a given pair breaking parameter $\alpha .^{46,47}$ The latter we interpolate as $\alpha=0.5 \Delta_{0}^{*}\left(B / B_{\mathrm{c}}\right)^{n}$, with $n$ a characteristic exponent. As shown in Fig. 2(e), the best fit we obtain for $n=3, \Delta_{0}^{*}=1.12 \mathrm{meV}$ and the (upper) critical field $B_{\mathrm{c}}=14.5 \mathrm{~T}$. The latter value is clearly larger than reported for bulk MoRe. Seemingly similar data plotted as purple stars and blue rectangles we discuss below.

As expected for a superconducting energy gap, $\Delta^{*}$ is also reduced with increasing temperature $T$. In Fig. 2(f) we plot $G_{23}$ as a function of $V_{\mathrm{SD}}$ at $V_{\mathrm{BG}}=9 \mathrm{~V}$ for a series of temperatures at $B=0$ in a different cooldown. For the lowest values of $T$, we can reproduce the data using very similar BTK and Dynes parameters as above, only adjusting the normal state resistance and the temperature to $T=1.7 \mathrm{~K}$. However, at higher temperatures, the fits become ambiguous, due to a broadening and possibly a temperature dependence of the Schottky barrier. ${ }^{30}$ Again, we plot the inflection points of the curves as an estimate for $\Delta^{*}$, as shown in Fig. $2(\mathrm{~g})$. To determine the critical temperature, we fit the expression $\Delta^{*}=\Delta_{0}^{*} \sqrt{1-T / T_{\mathrm{c}}}$ and find $T_{\mathrm{c}}=7.7 \mathrm{~K}$ and $\Delta^{*}=1.2 \mathrm{meV}$, consistent with literature values for bulk MoRe. ${ }^{38,39}$

Up to this point, our experiments demonstrate superconducting contacts, but with a weak electronic coupling between the $\mathrm{MoS}_{2}$ and the reservoirs, at least for one contact junction. However, 

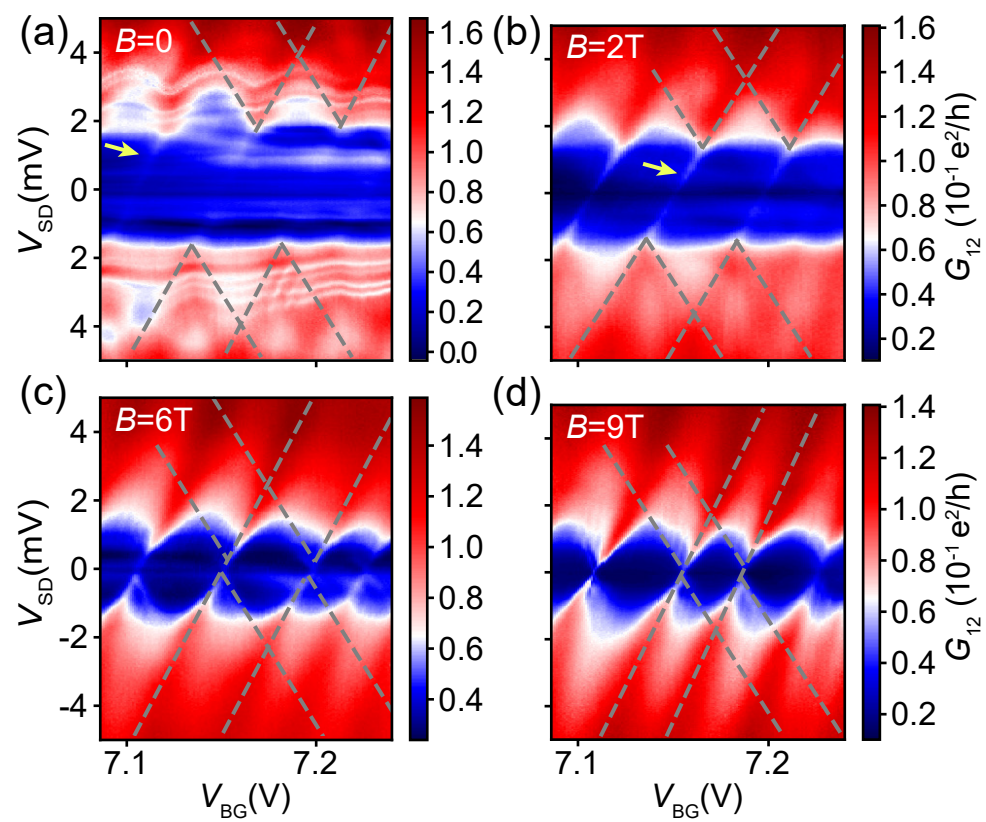

Figure 3: $G_{12}$ plotted as a function of $V_{\mathrm{BG}}$ and $V_{\mathrm{SD}}$ at (a) $B=0$, (b) $B=2 \mathrm{~T}$, (c) $B=6 \mathrm{~T}$ and (d) $B=9 \mathrm{~T}$, recorded at $T=60 \mathrm{mK}$. The dashed lines trace the $\mathrm{CB}$ diamonds, shifted vertically and horizontally between the subfigures, while the yellow arrows point out lines of resonant Andreev tunneling.

we also find evidence for a stronger coupling of $\mathrm{MoS}_{2}$ regions to the superconductors, relevant for devices relying on the superconducting proximity effect. As an example, we show bias spectroscopy measurements with $\mathrm{CB}$ features between contacts $\mathrm{C}_{1}$ and $\mathrm{C}_{2}$ in Fig. 3, for a series of magnetic fields $B$. Similarly as in Fig. 2, we find a transport gap, reduced by larger $B$ values. Here, the low-bias ends of the CB diamonds are shifted in energy and in gate voltage, as indicated by the gray dashed lines, consistent with a $\mathrm{MoS}_{2}$ quantum dot (QD) directly coupled to one superconducting contact (i.e. forming an S-QD-N junction). ${ }^{48}$ These tips of the CB diamonds are connected across the gap by a single faint resonance, pointed out by yellow arrows, best seen in Fig. 3(b) at $B=2 \mathrm{~T}$. We attribute these lines to resonant Andreev tunneling, ${ }^{48}$ in which the electrons of a Cooper pair pass through the QD in a higher order tunneling process. This process is suppressed much stronger by a tunnel barrier than single particle tunneling, ${ }^{49}$ which suggests that the QD is strongly coupled to the superconductor. At large $B$ fields, a quasi-particle background in the superconducting density of states results in single particle CB diamonds. ${ }^{42}$ With a QD charging energy of $\sim 2 \mathrm{meV}$ and using $E_{\mathrm{c}}=\frac{e^{2}}{8 \varepsilon \varepsilon_{0} r}$ for a disc shaped QD encapsulated in hBN, we estimate 
the radius of the confined QD region as $r \approx 300 \mathrm{~nm}$.

The shift of the $\mathrm{CB}$ diamonds in $V_{\mathrm{SD}}$ gives a measure for $\Delta^{*},{ }^{48}$ which we read out at the bias at which $\sim 50 \%$ of the large bias conductance is reached at the tip of the CB diamond. The extracted values are plotted as purple stars in Fig. 2(e). Surprisingly, we find a significantly larger zero field gap, $\Delta_{0}^{*} \approx 1.7 \mathrm{meV}$, and a rather different functional dependence on $B$ than for the curves analyzed in Fig. 2 (red dots). The latter is demonstrated by the dotted line obtained for the exponent $n=2$, and $B_{\mathrm{c}}=6.4 \mathrm{~T}$. In addition, Fig. 2(e) shows a third $\Delta^{*}$ curve extracted from CB diamond shifts in experiments on another contact pair shown in the supplemental data, Fig. S1. For this curve, we obtain $n=1$, while $\Delta^{*} \approx 1.12 \mathrm{meV}$ and $B_{\mathrm{c}} \approx 12 \mathrm{~T}$ correspond well to the previously obtained values.

While a larger gap in the transport experiments can be simply attributed to a significant fraction of the bias developing across another part of the device, for example across the second N/S junction, the different functional dependence is more difficult to explain. Since nominally the geometry and MoRe film thickness are identical for all contacts, we tentatively attribute this finding to a superconducting proximity region forming in the $\mathrm{MoS}_{2}$ near a strongly couped contact, yielding an induced superconducting energy gap $\Delta^{*}, 42$ and a different $B$-field dependence of the pair breaking compared to the bulk superconductor.

Additional indications for a stronger coupling to $\mathrm{S}$ are the almost gate voltage independent features at $B=0$, reminiscent of two NS junctions and multiple Andreev reflection processes in between, with a much stronger suppression with increasing $B$ than for the observed gap. In the supplemental data, Fig. S2(a), we also show data at higher gate voltages, exhibiting a conductance minimum instead of a maximum at the bias that corresponds to the energy gap, developing into negative differential conductance at the lowest field values. These findings are qualitatively consistent with calculations for an $\mathrm{S} / \mathrm{I} / \mathrm{N} / \mathrm{S}$ junction with resonances in the $\mathrm{N}$ region. ${ }^{50}$

The above data show that our fabrication scheme results in superconducting contacts to monolayer $\mathrm{MoS}_{2}$, possibly with a reasonably strong coupling to the superconductors for some of the contacts. To demonstrate that the intrinsic properties of $\mathrm{MoS}_{2}$ are intact in the bulk crystal, we in- 

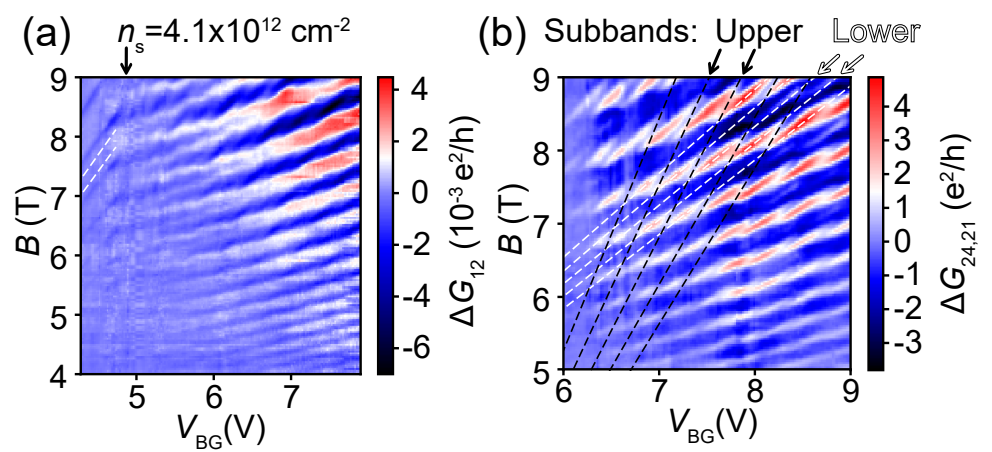

Figure 4: (a) Two-terminal dc conductance $G_{12}=I_{1} / V_{\mathrm{SD}}$ with $V_{\mathrm{SD}}=8 \mathrm{mV}$ applied to contact $\mathrm{C}_{2}$, plotted as a function of the magnetic field $B$ and the backgate voltage $V_{\mathrm{BG}}$ at $T \approx 60 \mathrm{mK}$. $n_{\mathrm{s}}$ points out the gate voltage corresponding to the electron density at which the higher spin-orbit subbands start to be populated. (b) Three-terminal dc conductance $G_{24,21}=I_{2} / V_{12}$, with an external bias $V_{\mathrm{SD}}=10 \mathrm{mV}$ applied to $\mathrm{C}_{4}$, while the current $I_{2}$ is measured at $\mathrm{C}_{2}$ and the voltage difference $V_{12}$ between $\mathrm{C}_{1}$ and $\mathrm{C}_{2}$. In both maps, a third order polynomial was subtracted at each gate voltage to remove a smooth background.

vestigate quantum transport in large magnetic fields and at bias voltages large enough to render the superconducting energy gap irrelevant. In Fig. 4(a) we plot the dc conductance $G_{12}=I_{1} / V_{\mathrm{SD}}$ as a function of $V_{\mathrm{BG}}$ and $B$, at $V_{\mathrm{SD}}=8 \mathrm{mV}$ and $T=60 \mathrm{mK}$, from which we have subtracted a third order polynomial background for each gate voltage to eliminate effects from the classical Hall effect and CB effects.

Figure 4(a) shows well developed Shubnikov de Haas $(\mathrm{SdH})$ oscillations, suggesting a high $\mathrm{MoS}_{2}$ quality, with an onset at $B_{\mathrm{on}}<4 \mathrm{~T}$. In the Drude model, this onset is interpreted as the charge carriers closing a cyclotron orbit before being scatted, which happens roughly at $\omega_{\mathrm{c}} \tau>1$, with $\omega_{\mathrm{c}}=e B / m^{*}$ the cyclotron frequency, $m^{*}$ the effective electron mass, and $\tau$ the scattering time. This yields a lower bound for the carrier mobility of $\mu=e \tau / m^{*} \approx 2500 \mathrm{~cm}^{2} /(\mathrm{Vs})$, similar to $\mu \approx 5000 \mathrm{~cm}^{2} /(\mathrm{Vs})$ we obtain with Au VIA contacts, identical to the best literature values. ${ }^{24}$ The discrepancy in mobility between the MoRe and the Au VIA contacts we attribute to heating effects due to the much larger bias we apply to the MoRe contacts to avoid effects of the superconducting energy gap.

The quality of the $\mathrm{MoS}_{2}$ can also be seen in the fact that the the four lowest spin-orbit subbands, corresponding to the valley and the spin degree of freedom, are not mixed by disorder. We find that 
the slope of the $\mathrm{SdH}$ resonances changes by roughly a factor of two at $V_{\mathrm{BG}}^{*}=4.8 \mathrm{~V}$, corresponding to an electron density of $n_{\mathrm{s}} \approx 4.1 \times 10^{12} \mathrm{~cm}^{-2}$, at which the two upper spin-orbit subbands become populated. Using $m^{*}=0.6$, we obtain a subband spacing of $\sim 15 \mathrm{meV}$, as reported previously. ${ }^{23}$ These features prevail also at $T \approx 1.7 \mathrm{~K}$, as shown in the supplemental data, Fig. S2(b).

The two-terminal magneto-conductance measurements suffer from large background resistances due to Shottky barriers, which we can partially circumnavigate by performing a three terminal experiment. In Fig. 4(b) we plot the dc conductance $G_{24,21}$, as explained in the figure caption. This technique removes the contact resistance at $\mathrm{C} 4$, so that the conductance resonances due to the Landau levels can be measured more clearly. The results in Fig. 4(b) show similar patterns as in better suited Hall bar experiments, ${ }^{23}$ exhibiting clear superposition patterns of the spin and valley split subbands, indicated by dashed lines. We note that due to the less ideal contact geometry of our devices, we cannot go to lower electron densities in these experiments, because the current density passing near the remote contacts is very low.

In conclusion, we established superconducting contacts to a monolayer of the TMDC semiconductor $\mathrm{MoS}_{2}$ using vertical interconnect access (VIA) contacts, and characterized the superconducting energy gap in different transport regimes. The fact that in most experiments one N/S junction dominates the transport characteristics, and signatures of resonant Andreev tunneling and a superconducting proximity effect, suggest that also contacts with a stronger transmission between the superconductor and the semiconductor are possible, thus opening a path towards semiconductor-superconductor hybrid devices at the limit of miniaturization, with a group of materials - the TMDCs - that offers a very large variety of material properties and physical phenomena.

\section{Associated content}

The supplemental data mentioned in the text as Figs. S1 and S2 are available free of charge on the ACS Publications website at DOI: 
All data in this publication are available in numerical form at doi.org/10.5281/zenodo.4518683

\section{Acknowledgments}

This work was supported financially by the Swiss National Science Foundation project "Twodimensional semiconductors for superconductor hybrid nanostructures" granted to AB, the Swiss Nanoscience Institute (SNI) project P1701, and the ERC project Top-Supra (787414). K.W. and T.T. acknowledge support from the Elemental Strategy Initiative conducted by the MEXT, Japan, Grant Number JPMXP0112101001, JSPS KAKENHI Grant Numbers JP20H00354 and the CREST (JPMJCR15F3), JST.

\section{References}

(1) Larsen, T. W.; Petersson, K. D.; Kuemmeth, F.; Jespersen, T. S.; Krogstrup, P.; Nygård, J.; Marcus, C. M. Semiconductor-nanowire-based superconducting qubit. Phys. Rev. Lett. 2015, $115,127001$.

(2) Leivo, M.; Pekola, J.; Averin, D. Efficient Peltier refrigeration by a pair of normal metal/insulator/superconductor junctions. Appl. Phys. Lett. 1996, 68, 1996.

(3) Roddaro, S.; Pescaglini, A.; Ercolani, D.; Sorba, L.; Giazotto, F.; Beltram, F. Hot-electron effects in InAs nanowire Josephson junctions. Nano Research 2011, 4, 259.

(4) Mourik, V.; Zuo, K.; Frolov, S. M.; Plissard, S.; Bakkers, E. P.; Kouwenhoven, L. P. Signatures of Majorana fermions in hybrid superconductor-semiconductor nanowire devices. Science 2012, 336, 1003.

(5) Deng, M.; Vaitiekėnas, S.; Hansen, E. B.; Danon, J.; Leijnse, M.; Flensberg, K.; Nygård, J.; Krogstrup, P.; Marcus, C. M. Majorana bound state in a coupled quantum-dot hybridnanowire system. Science 2016, 354, 1557. 
(6) Hofstetter, L.; Csonka, S.; Nygård, J.; Schönenberger, C. Cooper pair splitter realized in a two-quantum-dot Y-junction. Nature 2009, 461, 960.

(7) Hofstetter, L.; Csonka, S.; Baumgartner, A.; Fülöp, G.; d'Hollosy, S.; Nygård, J.; Schönenberger, C. Finite-bias Cooper pair splitting. Phys. Rev. Lett. 2011, 107, 136801.

(8) Fülöp, G.; d’Hollosy, S.; Baumgartner, A.; Makk, P.; Guzenko, V.; Madsen, M.; Nygård, J.; Schönenberger, C.; Csonka, S. Local electrical tuning of the nonlocal signals in a Cooper pair splitter. Phys. Rev. B 2014, 90, 235412.

(9) Wan, Z.; Kazakov, A.; Manfra, M. J.; Pfeiffer, L. N.; West, K. W.; Rokhinson, L. P. Induced superconductivity in high-mobility two-dimensional electron gas in gallium arsenide heterostructures. Nat. Commun. 2015, 6, 7426.

(10) Casparis, L.; Connolly, M. R.; Kjaergaard, M.; Pearson, N. J.; Kringhøj, A.; Larsen, T. W.; Kuemmeth, F.; Wang, T.; Thomas, C.; Gronin, S., et al. Superconducting gatemon qubit based on a proximitized two-dimensional electron gas. Nat. Nanotechnol. 2018, 13, 915.

(11) Fornieri, A.; Whiticar, A. M.; Setiawan, F.; Portolés, E.; Drachmann, A. C.; Keselman, A.; Gronin, S.; Thomas, C.; Wang, T.; Kallaher, R., et al. Evidence of topological superconductivity in planar Josephson junctions. Nature 2019, 569, 89.

(12) Vischi, F.; Carrega, M.; Braggio, A.; Paolucci, F.; Bianco, F.; Roddaro, S.; Giazotto, F. Electron Cooling with Graphene-Insulator-Superconductor Tunnel Junctions for Applications in Fast Bolometry. Phys. Rev. Appl. 2020, 13, 054006.

(13) Graziano, G. V.; Lee, J. S.; Pendharkar, M.; Palmstrøm, C. J.; Pribiag, V. S. Transport studies in a gate-tunable three-terminal Josephson junction. Phys. Rev. B 2020, 101, 054510.

(14) Xiao, D.; Liu, G.-B.; Feng, W.; Xu, X.; Yao, W. Coupled spin and valley physics in monolayers of MoS 2 and other group-VI dichalcogenides. Phys. Rev. Lett. 2012, 108, 196802. 
(15) Bhowal, S.; Satpathy, S. Intrinsic orbital and spin Hall effects in monolayer transition metal dichalcogenides. Phys. Rev. B 2020, 102, 035409.

(16) Žutić, I.; Fabian, J.; Sarma, S. D. Spintronics: Fundamentals and applications. Rev. Mod. Phys. 2004, 76, 323.

(17) Wang, Q. H.; Kalantar-Zadeh, K.; Kis, A.; Coleman, J. N.; Strano, M. S. Electronics and optoelectronics of two-dimensional transition metal dichalcogenides. Nat. Nanotechnol. 2012, 7, 699.

(18) Kormányos, A.; Zólyomi, V.; Drummond, N. D.; Burkard, G. Spin-orbit coupling, quantum dots, and qubits in monolayer transition metal dichalcogenides. Phys. Rev. X 2014, 4, 011034.

(19) Qiu, D. Y.; Felipe, H.; Louie, S. G. Optical spectrum of MoS 2: many-body effects and diversity of exciton states. Phys. Rev. Lett. 2013, 111, 216805.

(20) Kormányos, A.; Burkard, G.; Gmitra, M.; Fabian, J.; Zólyomi, V.; Drummond, N. D.; Fal'ko, V. k· p theory for two-dimensional transition metal dichalcogenide semiconductors. 2D Mater. 2015, 2, 022001.

(21) Tang, S.; Zhang, C.; Wong, D.; Pedramrazi, Z.; Tsai, H.-Z.; Jia, C.; Moritz, B.; Claassen, M.; Ryu, H.; Kahn, S., et al. Quantum spin Hall state in monolayer 1T'-WTe 2. Nat. Phys. 2017, 13,683 .

(22) Miserev, D.; Klinovaja, J.; Loss, D. Exchange intervalley scattering and magnetic phase diagram of transition metal dichalcogenide monolayers. Phys. Rev. B 2019, 100, 014428.

(23) Pisoni, R.; Kormányos, A.; Brooks, M.; Lei, Z.; Back, P.; Eich, M.; Overweg, H.; Lee, Y.; Rickhaus, P.; Watanabe, K., et al. Interactions and magnetotransport through spin-valley coupled Landau levels in monolayer MoS 2. Phys. Rev. Lett. 2018, 121, 247701.

(24) Pisoni, R.; Lei, Z.; Back, P.; Eich, M.; Overweg, H.; Lee, Y.; Watanabe, K.; Taniguchi, T.; 
Ihn, T.; Ensslin, K. Gate-tunable quantum dot in a high quality single layer MoS2 van der Waals heterostructure. Appl. Phys. Lett. 2018, 112, 123101.

(25) Marega, G. M.; Zhao, Y.; Avsar, A.; Wang, Z.; Tripathi, M.; Radenovic, A.; Kis, A. Logicin-memory based on an atomically thin semiconductor. Nature 2020, 587, 72-77.

(26) Island, J. O.; Steele, G. A.; van der Zant, H. S.; Castellanos-Gomez, A. Thickness dependent interlayer transport in vertical MoS2 Josephson junctions. 2D Mater. 2016, 3, 031002.

(27) Trainer, D. J.; Wang, B.; Bobba, F.; Samuelson, N.; Xi, X.; Zasadzinski, J.; Nieminen, J.; Bansil, A.; Iavarone, M. Proximity-Induced Superconductivity in Monolayer MoS2. ACS nano 2020, 14, 2718.

(28) Costanzo, D.; Jo, S.; Berger, H.; Morpurgo, A. F. Gate-induced superconductivity in atomically thin MoS 2 crystals. Nat. Nanotechnol. 2016, 11, 339.

(29) Roch, J. G.; Froehlicher, G.; Leisgang, N.; Makk, P.; Watanabe, K.; Taniguchi, T.; Warburton, R. J. Spin-polarized electrons in monolayer MoS 2. Nat. Nanotechnol. 2019, 14, 432.

(30) Cui, X.; Lee, G.-H.; Kim, Y. D.; Arefe, G.; Huang, P. Y.; Lee, C.-H.; Chenet, D. A.; Zhang, X.; Wang, L.; Ye, F., et al. Multi-terminal transport measurements of MoS 2 using a van der Waals heterostructure device platform. Nat. Nanotechnol. 2015, 10, 534.

(31) Allain, A.; Kang, J.; Banerjee, K.; Kis, A. Electrical contacts to two-dimensional semiconductors. Nat. Matt. 2015, 14, 1195-1205.

(32) Schauble, K.; Zakhidov, D.; Yalon, E.; Deshmukh, S.; Grady, R. W.; Cooley, K. A.; McClellan, C. J.; Vaziri, S.; Passarello, D.; Mohney, S. E.; Toney, M. F.; Sood, A.; Salleo, A.; Pop, E. Uncovering the Effects of Metal Contacts on Monolayer MoS2. ACS Nano 2020, 14, $14798-14808$.

(33) Samm, J.; Gramich, J.; Baumgartner, A.; Weiss, M.; Schönenberger, C. Optimized fabrication and characterization of carbon nanotube spin valves. J. Appl. Phys. 2014, 115, 174309. 
(34) Lembke, D.; Bertolazzi, S.; Kis, A. Single-layer MoS2 electronics. Acc. Chem. Res. 2015, $48,100$.

(35) Seredinski, A.; Arnault, E.; Costa, V.; Zhao, L.; Larson, T.; Watanabe, K.; Taniguchi, T.; F.Amet,; Newaz, A.; ; Finkelstein, G. One-Dimensional Edge Contact to Encapsulated MoS2 with a Superconductor. arXiv: 2021, 2101.06194.

(36) Telford, E. J.; Benyamini, A.; Rhodes, D.; Wang, D.; Jung, Y.; Zangiabadi, A.; Watanabe, K.; Taniguchi, T.; Jia, S.; Barmak, K., et al. Via method for lithography free contact and preservation of 2D Mater. Nano Lett. 2018, 18, 1416.

(37) Witcomb, M.; Dew-Hughes, D. The $\sigma$-phase in molybdenum-rhenium; microstructure and superconducting hysteresis. J. Less-Common Met. 1973, 31, 197.

(38) Sundar, S.; Sharath Chandra, L.; Sharma, V.; Chattopadhyay, M.; Roy, S. Electrical transport and magnetic properties of superconducting Mo 52 Re 48 alloy. AIP Conference Proceedings. 2013; pp 1092-1093.

(39) Singh, V.; Schneider, B. H.; Bosman, S. J.; Merkx, E. P.; Steele, G. A. Molybdenum-rhenium alloy based high-Q superconducting microwave resonators. Appl. Phys. Lett. 2014, 105, 222601.

(40) Pillet1, J.-D.; Quay, C. H. L.; Morfin, P.; Bena, C.; Yeyati, A. L.; Joyez, P. Andreev bound states in supercurrent-carrying carbon nanotubes revealed. Nat. Physics 2010, 6, 965.

(41) Gramich, J.; Baumgartner, A.; Schönenberger, C. Andreev bound states probed in threeterminal quantum dots. Phys. Rev. B 2017, 96, 195418.

(42) Jünger, C.; Baumgartner, A.; Delagrange, R.; Chevallier, D.; Lehmann, S.; Nilsson, M.; Dick, K. A.; Thelander, C.; Schönenberger, C. Spectroscopy of the superconducting proximity effect in nanowires using integrated quantum dots. Commun. Phys. 2019, 2, 1. 
(43) Heedt, S.; Quintero-Pérez, M.; Borsoi, F.; Fursina, A.; van Loo, N.; Mazur, G. P.; Nowak, M. P.; Ammerlaan, M.; Li, K.; Korneychuk, S., et al. Shadow-wall lithography of ballistic superconductor-semiconductor quantum devices. arXiv preprint arXiv:2007.14383 2020,

(44) Blonder, G.; Tinkham, M.; Klapwijk, T. Transition from metallic to tunneling regimes in superconducting microconstrictions: Excess current, charge imbalance, and supercurrent conversion. Phys. Rev. B 1982, 25, 4515.

(45) Dynes, R.; Narayanamurti, V.; Garno, J. P. Direct measurement of quasiparticle-lifetime broadening in a strong-coupled superconductor. Phys. Rev. Lett. 1978, 41, 1509.

(46) Skalski, S.; Betbeder-Matibet, O.; Weiss, P. Properties of Superconducting Alloys Containing Paramagnetic Impurities. Phys. Rev. 1964, 136, A1500.

(47) Gramich, J.; Baumgartner, A.; Schönenberger, C. Subgap resonant quasiparticle transport in normal-superconductor quantum dot devices. Appl. Phys. Lett. 2016, 108, 172604.

(48) Gramich, J.; Baumgartner, A.; Schönenberger, C. Resonant and inelastic Andreev tunneling observed on a carbon nanotube quantum dot. Phys. Rev. Lett. 2015, 115, 216801.

(49) Sun, Q.-f.; Wang, J.; Lin, T.-h. Resonant Andreev reflection in a normal-metal-quantum-dotsuperconductor system. Phys. Rev. B 1999, 59, 3831.

(50) Zhitlukhina, E.; Devyatov, I.; Egorov, O.; Belogolovskii, M.; Seidel, P. Anomalous inner-gap structure in transport characteristics of superconducting junctions with degraded interfaces. Nanoscale Res. Lett. 2016, 11, 1. 